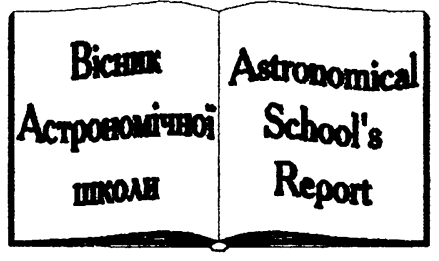

\title{
$\begin{array}{ll}\text { Tом } 1 \cdot \text { № } 1 \cdot 2000 & \text { C. } 34-43\end{array}$
}

UDC 521; 528

\section{Solutions of Clairaut's equation and the piecewise Roche's density model a)}

\section{A.N. Marchenko}

State University "Lviv Polytechnic", Lviv, Ukraine

SOLUTIONS OF CLAIRAUT'S EQUATION AND THE PIECEWISE ROCHE'S DENSITY MODEL, by Marchenko A.N. - The piecewise Roche's model is considered as one of the possible solutions (exact or approximate) of Clairaut, Poisson, and Williams-Adams equations. Necessary theoretical aspects were considered before the numerical investigations, which based on the fundamental constants of geodesy and astronomy together with global data of the seismic radial tomography of the Earth's interior.

РОЗВ'ЯЗКИ РІВНЯННЯ КЛЕРО ТА КУСКОВО-НЕПЕРЕРВНА МОДЕЛЬ РОША РАДІАЛЬНОГО РОЗПОДІЛУ ГУСТИНИ, Марченко О.М. - Кусково-неперервна модель Роша розглядається як один з можливих розв'язків (точних або наближених) рівнянь Клеро, Пуасона та Вільямсона-Адамса. Розглянуто необхідні теоретичні аспекти проблеми та виконане чисельне дослідження, що базується на фундаментальних сталих геодезії та астрономіі разом 3 глобальними даними радіальної сейсмічної томографії Землі.

РЕШЕНИЯ УРАВНЕНИЯ КЛЕРО И КУСОЧНО-НЕПРЕРЫВНАЯ МОДЕЛЬ РОША РАДИАЛЬНОГО РАСПРЕДЕЛЕНИЯ ПЛОТНОСТИ, Марченко А.Н. - Кусочно-непрерывная модель Роша рассматривается как одно из возможных решений (точных либо приближенньх) уравнений Клеро, Пуассона и Вильямсона-Адамса. Рассмотрены необходимые теоретические аспекты проблемы и выполнено численное исследование, которое основано на фундаментальных постоянных геодезии и астрономии вместе с глобальньми данньми радиальной сейсмической томографии Земли.

Starting from the first investigations of the Earth's density distribution some remarkable and simple density laws were constructed by Legendre, Laplace, Darwin, Roche, etc. These laws have a spherically symmetric density distribution with the volume density $\rho(\ell)$ that depends on the radial distance $\ell$. At the Geodetic Week97 (Berlin,1977) the author had several interesting discussions with Prof. E.Grafarend about the exponential nature of the flattening distribution according to the Clairaut's equation. Maybe these meetings and discussions yielded now the presented consideration of the famous classic laws of density in view of their mathematical descriptions. As a result, the latter is the main goal of this paper. At present one of "the simplest way to represent an smoothed density law is

a) Part of this paper was presented into Festschrift to $60^{\text {th }}$ anniversary celebration of Professor E. Grafarend. 
by means of a polynomial" (Moritz,1990) e.g. in accordance with the piecewise PREM model for every segment after the suitable stratification of the Earth. Note that such a representation follows, for instance, from the traditional requirement to the Earth's standard model (Dziewonski and Andeson, 1981) "... for each regions, a form of a low-degree polynomial in radius". In spite of such "standard" approach, the other goal of this paper is the creation of the piecewise Roche's model. At a first look such model has only even degrees with the maximal second degree. Nevertheless the piecewise Roche's model is considered here as one of the possible solutions (exact or approximate) of Clairaut, Poisson, and Williams-Adams equations. Some theoretical aspects were considered before the numerical investigations, which based on the fundamental constants of geodesy and astronomy together with global data of the seismic radial tomography of the Earth's interior.

\section{BASIC RELATIONSHIPS}

In view of a mathematical formulation the traditional representation of the Earth's radial density can be treated as a function $\rho(\ell)$ (continuos or piecewise in form of shells) of one variable $\ell$ only, which is defined on the finite segment $(0 \leq \ell \leq R)$ only if we assume that the figure of the planet is spherical, ( $R$ is the mean Earth's radius $(R=6371 \mathrm{~km})$ ). It is well-known also (Moritz, 1990) that in this case the gravitational potential $V$ is equal to the gravity potential $W$, since we use such simplest approximation of the ellipsoid by the sphere when the flattening $f=0$.

First of all our initial (observed) information will be the Earth's mass $M$ and the mean moment of inertia $I$. For latter use we shall write some well-known formulae within the sphere of the radius $\ell$ (the part of the Earth's mass which is restricted by this radius) for the mass

$$
M(\ell)=4 \pi \int_{0}^{\ell} \rho(x) x^{2} d x,
$$

where $d x$ is the element of a line and the mean density $D(\ell)$ :

$$
D(\ell)=\frac{3}{4 \cdot \pi \cdot \ell^{3}} M(\ell) .
$$

The value $D(\ell)$ in the form of (2) leads to the following representations

$$
g(\ell)=\frac{4 \cdot \pi \cdot G}{3} \ell \cdot D(\ell) \Leftrightarrow g(\ell)=\frac{G M}{\ell^{2}},
$$

of the gravity $\mathrm{g}(\ell)$ inside the Earth, where $\mathrm{G}=6.673 \cdot 10^{-8}\left[\mathrm{~cm}^{3} \mathrm{~s}^{2} \mathrm{~g}^{-1}\right]$ is the gravitational constant. The mean moment of inertia is

$$
I(\ell)=\frac{8 \pi}{3} \int_{0}^{\ell} \rho(x) x^{4} d x .
$$

We shall use also the seismic velocities $V_{p}$ and $V_{s}$ in the form of the function

$$
\Phi=\Phi(\ell)=V(\ell)_{P}^{2}-\frac{4}{3} V(\ell)_{S}^{2}
$$

by applying their grid values in accordance with (Dziewonski and Anderson, 1981), which practically represents the results of seismic tomography of the Earth interior. 


\section{THE SIMPLEST SOLUTIONS OF CLAIRAUT'S EQUATION}

Now we recollect that the famous oldest hypothesis for the Earth's density distribution were proposed after solutions of Clairaut's equation for the flattening inside the Earth (see, for instance, Bullen, 1975; Moritz, 1990).

There exist (Bullen, 1975) three famous solutions of this equation for the density $\rho$. First one is Legandre - Laplace law

$$
\rho(x)=\rho_{0} \frac{\sin (\beta x)}{\beta x}=\rho_{0} \frac{\exp (\sqrt{-1} \beta x)-\exp (-\sqrt{-1} \beta x)}{2 \sqrt{-1} \beta x}, \beta=\text { const },
$$

where we apply the dimensionless "radius-vector"

$$
x=\frac{\ell}{R},
$$

regarding to $R ; \rho_{0}=$ const and may be considered here as the density at the origin.

The second one is Roche's law

$$
\rho(x)=\rho_{0}\left(1-K x^{2}\right)=a+b x^{2},
$$

where

$$
a=\rho_{0}>0 \text { and } b=\rho_{0} K<0 .
$$

Note now that Taylor series expansion of (6) (disregarding other higher powers of $x$ ) in view of mathematics leads to the similar expression:

$$
\rho(x)=\rho_{0}\left(1-\frac{\beta^{2}}{6} x^{2}\right)
$$

The third one is G. Darwin law

$$
\rho(x)=C \cdot x^{-n},
$$

where $C$ is a constant. His solution involves an ,assumption of the form for the law of the internal density of the planet and subsequent determination of the law of compressibility" (Darwin, 1884) . Clearly, the expression (11) represents the density with a singularity at the origin. G.Darwin noted already that case $n=0$ for the model (11) corresponds to the case of homogeneous density; for $n=3$ the Earth's mass $M$ will become infinite; for $n>3$ the mass $M$ must be assumed to be negative. As a result, we get the inequality $0<n<3$ which agrees with the determination $n=1.011$ (Bullen, 1975). Thus the expression (11) represents a power function.

\section{WILLIAMSON-ADAMS EQUATION}

The density $\rho$ may fulfil the so-called Williamson-Adams equation for each shell of the stratified Earth under the following assumptions: the Earth is globally in hydrostatic equilibrium; chemical composition and phase transformation are homogeneous in every shell; the temperature is adiabatic in each shell. Thus, if we have the observable seismic velocity (5), in view of the gravitational (3) and hydrostatic relationships

$$
\operatorname{grad}(\ell)=\rho(\ell) \cdot \operatorname{grad} V(\ell) \Rightarrow \frac{d p(\ell)}{d \ell}=-\rho(\ell) \cdot g(\ell),
$$

finally the Williamson-Adams equation can be written as 


$$
\frac{d \ln \rho(\ell)}{d \ell}=-\frac{g(\ell)}{\Phi(\ell)}
$$

where $p$ is the pressure inside the Earth. Thus (13) is a formula to derive the radial density distribution from the seismic velocity data, fulfilled under the assumptions listed above.

In order to use (13) we must first try to solve this equation and to express the observed seismic data by a suitable function of depth, separating the Earth into convenient shells. Traditionally we shall assume that the separation into shells has to be choice at those spheres, where discontinuities in the parameter $\Phi$ or in its derivative can be observed.

It is evident that the formal solution of (13) may be obtained after the integration of Williamson-Adams equation. The result is

$$
\rho(\ell)=\rho_{0} \exp \left(-\int_{0}^{\ell} \frac{g(x)}{\Phi(x)} d x\right),
$$

and we get the functional dependence for radial density as an exponential function. The right hand side of the expression (14) is unknown. For this reason, we shall apply instead of (14) the simplest approximating function

$$
\rho(\ell)=\rho_{0} \exp \left(-\gamma^{2} x^{2}\right), \gamma=\text { const },
$$

where the power 2 is the lowest power for which we may get a non-zero value $\Phi$ at the origin. Taylor expansion of (15) leads again to the Roche's model

$$
\rho(x)=\rho_{0}\left(1-\gamma^{2} x^{2}\right)=a+b x^{2},
$$

if we disregard other higher powers of $x$.

\section{POISSON'S EQUATION}

The density $\rho$ must fulfil the Poisson's equation for the gravity potential $W=V$ of the Earth. Using the spherical coordinates after simple manipulations we get for a radial layered Earth, that is for $\rho=\rho(\ell)$, in spherical approximation

$$
-\Delta V=4 \pi G \rho=\frac{d g}{d \ell}+\frac{2 g}{\ell}=M[g] .
$$

The operator

$$
M[]=\frac{d}{d \ell}+\frac{2}{\ell},
$$

is well-known in geodesy as Molodensky operator (see, for instance, Neyman, 1979) and it was introduced first for the basic boundary problem of geodesy in the next form

$$
M[T]=\frac{d T}{d r}+\frac{2 T}{r}=-\Delta g,
$$

where $T$ is the anomalous potential, $\Delta g$ is the gravity anomaly, $r$ is the radius-vector of an external point (the parameter $\ell$ represents the radius-vector of an internal point).

The expression (19) is used for the determination of $T$ on the ground of known gravity anomalies. In the expression (17) we have as unknown values both the density and gravity inside the Earth. Nevertheless, if the gravity $g$ is known we get a simple rule for the computation of radial density profile in accordance with Poisson's equation. If gravity is known approximately, we get one 
of the most important additional information for a stable creation of the density models. So, one of our next steps will connected with the gravity distribution inside the Earth.

\section{SOME REMARKS ON THE REGULAR DARWIN'S LAW}

If we want to avoid a singularity at the origin in (11), this function may be transform to the expression

$$
\rho(x)=C \cdot x^{-f(x)}=C \cdot \exp (-f(x) \ln x),
$$

where $f(x)$ is any suitable function. Such a function can represents a regular form of Darwin's law without a singularity at the origin with the possible expression for $f(x)$ in the form of special polynomial without first constant term (Marchenko and Lelgemann, 1997). The expression (20) may be considered as an exponential function.

Taking into account the relationships (14), (15) we may try to insert into (20) another function $f(x)=F(x) / \ln (x)$ (in particular, $\left.F(x)=\gamma^{2} x^{2}\right)$ that leads on the whole again to

$$
\rho(x)=C \cdot \exp (-F(x)) \text {, }
$$

the solution (14) of Williamson-Adams equation and to the considered case (15) in particular.

Note that the direct integration of (20) is impossible for mass (1), for moment of inertia (4), etc. The expression (21) in the form of (15):

$$
\rho(\ell)=\rho_{0} \exp \left(-\gamma^{2} x^{2}\right)
$$

admits according to (1) and (4) the next remarkable expressions for the mass

$$
M(\ell)=\frac{4 \pi \rho_{0} R^{3}}{\gamma^{2}}\left[\frac{\sqrt{\pi} \cdot \operatorname{erf}(\gamma \cdot x)}{4 \gamma}-\frac{x}{2 \rho_{0}} \rho(\ell)\right],
$$

and for the mean moment of inertia

$$
I(\ell)=\frac{8 \pi \rho_{0} R^{5}}{3 \gamma^{4}}\left[\frac{3 \sqrt{\pi} \cdot \operatorname{erf}(\gamma \cdot x)}{8 \gamma}-\frac{x}{4 \rho_{0}} \rho(\ell) \cdot\left(2 \gamma^{2} x^{2}+3\right)\right]=\frac{R^{2}}{\gamma^{2}}\left[M(\ell)-\frac{4 \pi \ell^{3}}{3} \rho(\ell)\right],
$$

where $\operatorname{erf}(z)$ is the integral of the Gaussian distribution from 0 to $z$ or the probability integral with the density distribution according to (22).

Thus we come to a remarkable result: one of the possible solutions of the Williamson-Adams equation is nothing else but the famous Gaussian distribution, which may be approximated by the Roche 's model, represented the solution of Clairaut's equation.

So, in spite of the difference between considered above various expressions for density, we come to their exponential nature on the whole. Roche's model we may treat now as a truncated Taylor series of them or as approximate solution of Williamson-Adams equation.

\section{SAIGEY'S THEOREM AND THE ROCHE'S MODEL}

According to the so-called Saigey theorem, the gravity $g(\ell)$ has a maximum inside the Earth. We shall use the Roche's model as a basic tool for next study. So that, it is necessary to find such a point(s), where the radial derivative $\frac{d g(\ell)}{d \ell}$ is equal to zero. As a result, for the stationary point(s) we get the well-known expression 


$$
\frac{d g(\ell)}{d \ell}=\frac{4 \pi G}{3}\left(D(\ell)+\ell \frac{d D(\ell)}{d \ell}\right)=4 \pi G\left(\rho(\ell)-\frac{2}{3} D(\ell)\right)=0 \Rightarrow \rho(\ell)=\frac{2}{3} D(\ell) .
$$

Now applying the Roche's model (8) or (16) to (25) we get immediately

$$
D(\ell)=a+\frac{3 \cdot b}{5}\left(\frac{\ell}{R}\right)^{2}=a+\frac{3 \cdot b}{5} x^{2},
$$

and the solution of (25) for the parameter $x$

$$
x=\frac{\ell}{R}=\frac{\sqrt{5} \cdot \sqrt{a}}{3 \cdot \sqrt{-b}} .
$$

Note that this root of (25) corresponds to (9) and $a>0$. In this case the sign of $b$ must be negative: $b<0$. Moreover applying such dimensionless $x \in[0,1]$ and (27) the following inequality

$$
\frac{a}{-b} \leq \frac{9}{5}
$$

may be found for the coefficients of the Roche's model. Note only that the sign of the second radial derivative follows from the coefficient $b$. For this reason $\frac{d^{2} g(\ell)}{d \ell^{2}}<0$ in the point (27) and our function $g(\ell)$ has a maximum only at this point.

\section{PIECEWISE ROCHE'S MODEL}

If a suitable stratification of the Earth leads to its division into $m$ shells, first we shall represent the density distribution by own Roche's model within every shell separately

$$
\rho_{i}(x)=a_{i}+b_{i} x^{2}, i=1,2, \ldots m \text {. }
$$

Inserting (29) into the expressions (1), (2), and (4) we get finally the recurrence formulae for the mass, the mean density and mean moment of inertia, respectively:

$$
\begin{gathered}
M_{1, m}(\ell)=M_{1, m-1}\left(\ell_{m-1}\right)+\left[M_{m}(\ell)-M_{m}\left(\ell_{m-1}\right)\right],\left(\ell_{m-1} \leq \ell \leq R\right), \\
D_{1, m}(\ell)=\left(\frac{\ell_{m-1}}{\ell}\right)^{3} D_{1, m-1}\left(\ell_{m-1}\right)+\left[D_{m}(\ell)-\left(\frac{\ell_{m-1}}{\ell}\right)^{3} D_{m}\left(\ell_{m-1}\right)\right], \\
I_{1, m}(\ell)=I_{1, m-1}\left(\ell_{m-1}\right)+\left[I_{m}(\ell)-I_{m}\left(\ell_{m-1}\right)\right],
\end{gathered}
$$

where for the piecewise Roche's model

$$
\begin{gathered}
M_{i}(\ell)=\frac{4 \pi}{3} \ell^{3}\left[a_{i}+\frac{3}{5} b_{i} x^{2}\right], M_{1,1}(\ell)=M_{1}(\ell), \\
D_{i}(\ell)=\left[a_{i}+\frac{3}{5} b_{i} x^{2}\right], D_{1,1}(\ell)=D_{1}(\ell), \\
I_{i}(\ell)=\frac{8 \pi}{3} \ell^{5}\left[\frac{a_{i}}{5}+\frac{b_{i}}{7} x^{2}\right], I_{1,1}(\ell)=I_{1}(\ell),
\end{gathered}
$$

starting from the first shell $\left(0 \leq \ell \leq \ell_{1}\right)$. In these formulae $\ell_{j}(j=1,2, \ldots m-1)$ are the fixed radiusvectors, where jumps of radial density are presupposed. The recurrence formulae for gravity is based on the expressions (3) and (31): 


$$
g_{1, m}(\ell)=\frac{4 \cdot \pi \cdot G}{3} \ell \cdot D_{1, m}(\ell),\left(\ell_{m-1} \leq \ell \leq R\right),
$$

again starting from the first shell.

For the recurrence formulae of the seismic parameter $\Phi$ and it jumps first we shall find

$$
\frac{d \ln \rho_{i}(\ell)}{d \ell}=\frac{2 b_{i} \ell}{R^{2} \rho_{i}(\ell)}
$$

Further by applying the Williamson-Adams equation (13) for the piecewise model (29) in view of (38) after some manipulations we get

$$
\begin{gathered}
\Phi_{1, m}(\ell)=-\frac{2 \cdot \pi \cdot G \cdot R^{2}}{3 \cdot b_{m}} \rho_{m}(\ell) \cdot D_{1, m}(\ell),\left(\ell_{m-1} \leq \ell \leq R\right), \\
\Phi_{i}(\ell)=-\frac{2 \cdot \pi \cdot G \cdot R^{2}}{3 \cdot b_{i}} \rho_{i}(\ell) \cdot D_{i}(\ell), \Phi_{1,1}(\ell)=\Phi_{1}(\ell),\left(0 \leq \ell \leq \ell_{1}\right) .
\end{gathered}
$$

By the definition (5) the parameter $\Phi$ must be always positive and we shall consider the ratio

$$
\frac{\Phi_{1, i}\left(\ell_{j-1}\right)}{\Phi_{1, i-1}\left(\ell_{j-1}\right)}=\frac{b_{i-1}}{b_{i}} \frac{\rho_{i}\left(\ell_{j-1}\right)}{\rho_{i-1}\left(\ell_{j-1}\right)}>0 \text {, }
$$

which must be positive for each boundary of two shells. From this inequality together with (9), (28) (for one shell) we come to a remarkable results: all coefficients $a_{i}$ will be positive and all coefficients $b_{i}$ will be negative for the piecewise Roche's model of density.

Finally we may compute the seismic jump of $\Phi$ at the $j$ - boundary

$$
\Delta \Phi=\Delta \Phi_{i, i+1}=\Phi_{i}\left(\ell_{j}\right)-\Phi_{i+1}\left(\ell_{j}\right)=-\frac{2 \cdot \pi \cdot G \cdot R^{2}}{3} D_{1, i}\left(\ell_{j}\right)\left[\frac{a_{i}}{b_{i}}-\frac{a_{i+1}}{b_{i+1}}\right] .
$$

This formula may use as the additional condition between the coefficients of every shell, because the left hand side of (41) is known from seismic data.

\section{PIECEWISE ROCHE'S SOLUTION FOR RADIAL DENSITY MODEL}

Now we recollect (see, for instance, Moritz, 1990) that „any global density law must satisfy three basic conditions:

1. It must provide the correct total mass or, equivalently, the mean density;

2. It must give the value for the mean moment of inertia;

3. It must reproduce the density at the base of continental layers, which may be taken as about 3.2 to $3.3 \mathrm{~g} / \mathrm{cm}^{3}$, e.g. the conventional density just below Mohorovichich discontinuity much used in isostasy $\rho_{1}=3.27 \mathrm{~g} / \mathrm{cm}^{3}$ “.

These three conditions may lead to the construction of the continuos radial density distribution. First two conditions can apply for the determination of the continuos Roche's model. In this case we get a remarkable expression for the coefficient $b$ of such a model

$$
\left.\begin{array}{c}
b=\frac{5}{3}\left[D-\rho_{0}\right] \\
a=\rho_{0}
\end{array}\right\} \text {. }
$$

Nevertheless, we may add according to (39) the additional condition for density at the origin, which will depend on the observe value of $\Phi$ : 


$$
\Phi(0)=-\frac{2 \cdot \pi \cdot G \cdot R^{2}}{3 \cdot b_{1}} a_{1}^{2}
$$

And use then four conditions for determinations of the coefficients of two $(M=2)$ models (29). We presuppose also that the first model will describe the density on the interval $[0,3480 \mathrm{~km}]$ and the second model is valid for the interval $[3480 \mathrm{~km}, 6371 \mathrm{~km}]$. Now according to three condition listed above we get

$$
\begin{gathered}
a_{1}\left(\frac{\ell_{1}}{R}\right)^{3}+a_{2}\left[1-\left(\frac{\ell_{1}}{R}\right)^{3}\right]+\frac{3 b_{1}}{5}\left(\frac{\ell_{1}}{R}\right)^{5}+\frac{3 b_{2}}{5}\left[1-\left(\frac{\ell_{1}}{R}\right)^{5}\right]=D, \\
\frac{2}{D}\left\{\frac{a_{1}}{5}\left(\frac{\ell_{1}}{R}\right)^{5}+\frac{a_{2}}{5}\left[1-\left(\frac{\ell_{1}}{R}\right)^{5}\right]+\frac{b_{1}}{7}\left(\frac{\ell_{1}}{R}\right)^{7}+\frac{b_{2}}{7}\left[1-\left(\frac{\ell_{1}}{R}\right)^{7}\right]\right\}=I_{d}, \\
a_{2}+b_{2}=\rho_{s},
\end{gathered}
$$

where $I_{d}=I / M R^{2}$ is the dimensionless Earth's moment of inertia, $\rho_{s}$ is the surface density, $D=D(R)$ is the Earth's mean density. Because the equation (43) is non-linear, on the first step we shall add the following linear equation

$$
a_{1}=\rho_{0},
$$

and will solve this system (44)-(47) with respect to the density (47) at the origin. On the second step the non-linear equation (43) may be solved numerically in a traditional way. After iterations, we can get these four coefficients and compute now the basic jump of the Earth's density. To our own surprise such solution of the equations (43)-(46) together with the seismic data alone provided finally (in this step) the density jump at the core/mantle boundary $\Delta \rho=4.454 \mathrm{~g} / \mathrm{cm}^{3}$, the density at the centre mass of the Earth $\rho=12.953 \mathrm{~g} / \mathrm{cm}^{3}$ (see Figure 1), and a remarkable stable restoring of the behavior of the gravity distribution (see Figure 3) corresponding to various number of shells of the Earth's stratification.

For this reason after the creation of these two models we may continue such approach for the further division of the Earth and determination of the set of the models (29) which should be agreed with the whole initial information about the seismic data.

Thus on the first step we may get a preliminary solution for every shell separately by the "golden section" technique (in view of the necessity of the Earth's stratification and solution of the non-linear equation (43)). The second step consists of the readjustment of these independent pieces of density to the piecewise density distribution which agrees with the set of the seismic data and other additional information about fundamental constants.

Regarding the discontinuities in the seismic velocities as sampled for PREM, we are led to the following separation into shells (Table 1) as a particular case. Based on this separation a mathematical description of the Earth's density based on the piecewise Roche's model was derived and presented in Table1. This model (see, Figure 2) can be used further for an improvement as a starting model using

Table 1. Piecewise Roche's density model $(m=7)$

\begin{tabular}{|c|c|c|c|c|}
\hline Shell & $a_{i}$ & $b_{i}$ & $\ell_{j}, \mathrm{~km}$ & $\begin{array}{c}\text { Density } \\
\text { jump }\end{array}$ \\
\hline 1 & 13.061 & -8.891 & 1221.5 & 0.558 \\
2 & 12.483 & -8.343 & 3480.0 & 4.392 \\
3 & 6.370 & -2.574 & 5701.0 & 0.314 \\
4 & 6.058 & -2.577 & 5971.0 & 0.228 \\
5 & 5.784 & -2.524 & 6151.0 & 0.080 \\
6 & 6.057 & -2.903 & 6346.6 & 0.476 \\
7 & 6.622 & -3.952 & \\
\hline
\end{tabular}


another - exponential or other solution of Williamson-Adams equation. Figure 2 reflects its good agreement with the PREM-density model, with the exception of the crust shells: we try to create on the final step a "geodetic version" of the Earth density profile with the surface density $\rho_{\mathrm{s}}=2.67 \mathrm{~g} / \mathrm{cm}^{3}$.

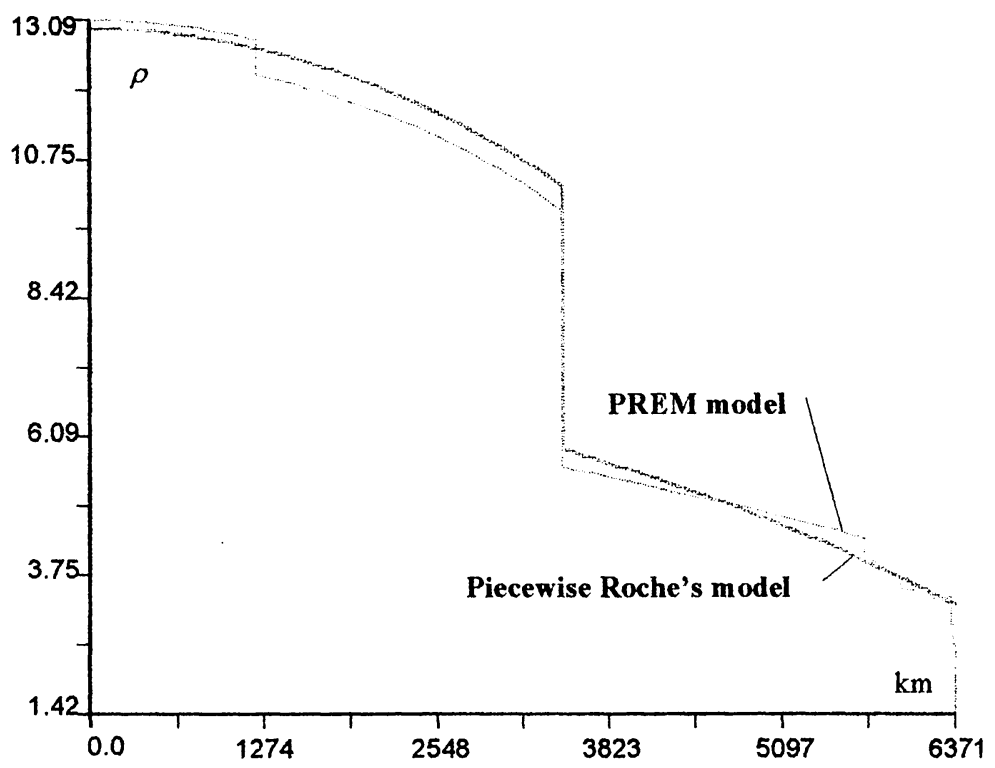

Figure 1. Comparison of the PREM-density $\rho\left[\mathrm{g} / \mathrm{cm}^{3}\right]$, with the twopiecewise density model.

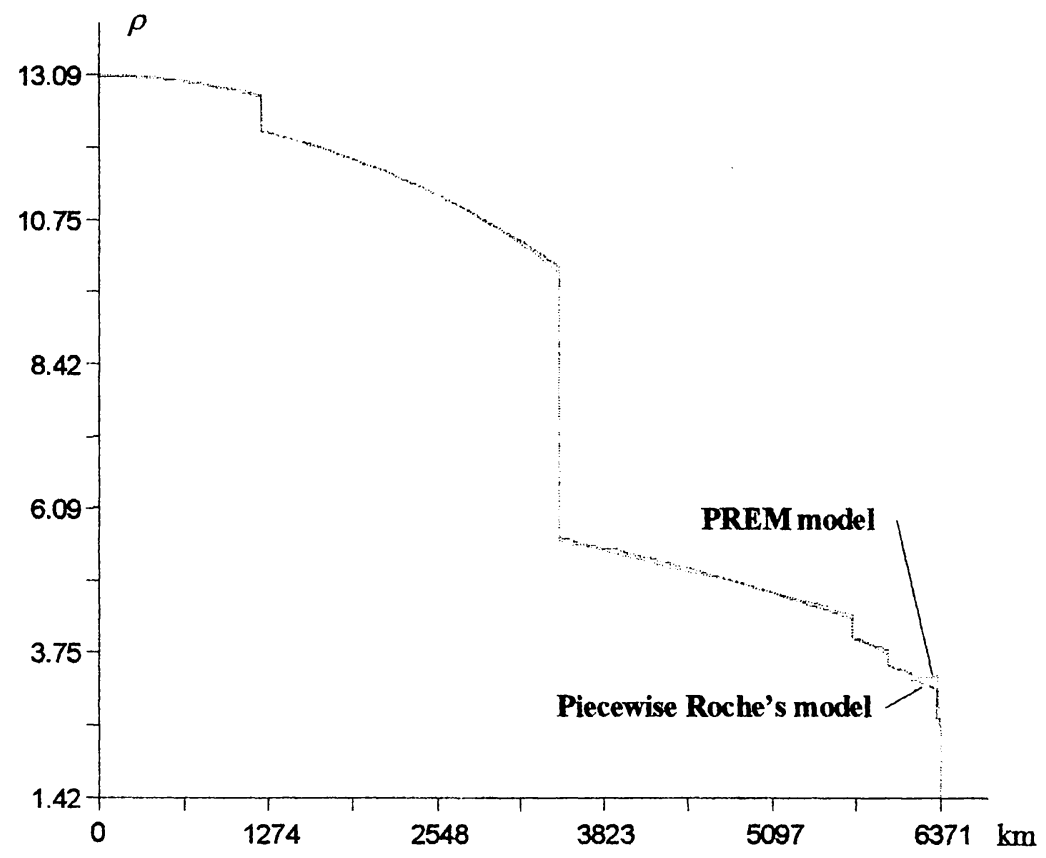

Figure 2. Comparison of the PREM-density $\rho\left[\mathrm{g} / \mathrm{cm}^{3}\right]$, with the final density model. 


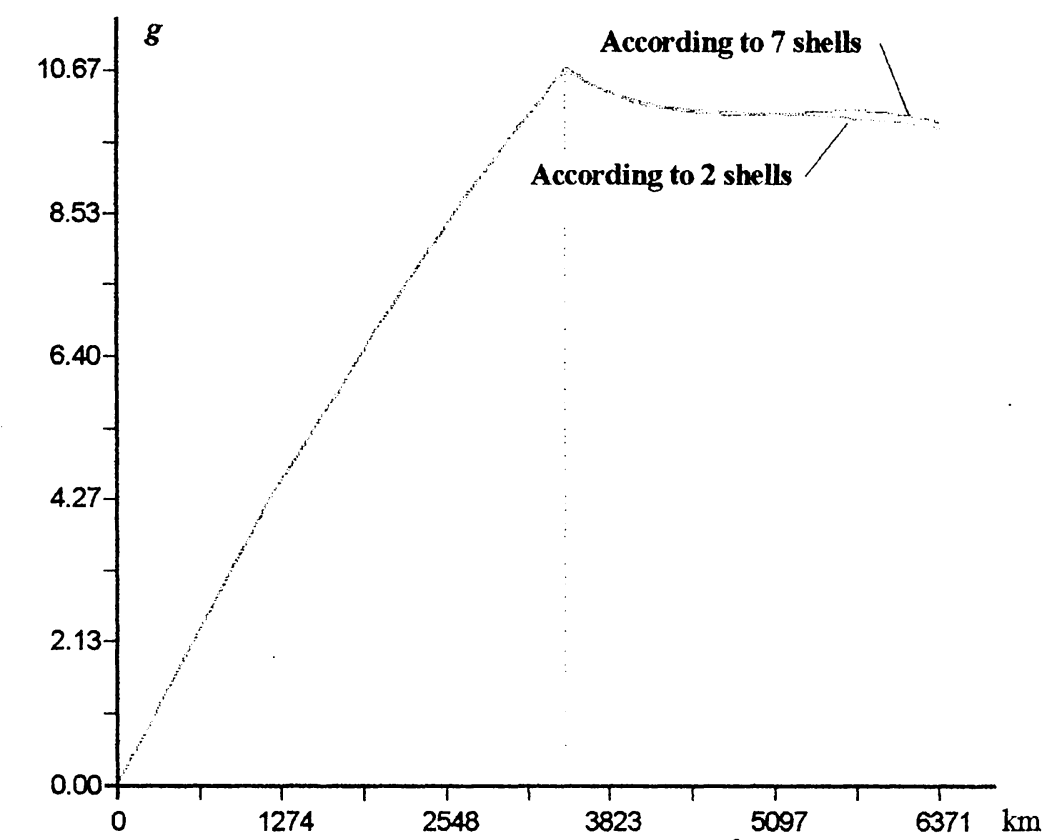

Figure 3. Comparison of the internal gravity $g\left[\mathrm{~m} / \mathrm{s}^{2}\right]$ distribution for various stratifications.

1. Bullen, K. E. (1975) The Earth's Density. Chapman and Hall, London.

2. Darwin, G. (1884) On the figure of equilibrium of a planet of heterogeneous density. Proceeding of the Royal Society, Vol. XXXVI, 158-166.

3. Dziewonski, A.M. and Anderson, D.L. (1981) Preliminary reference Earth model. Physics of the Earth and Planetary Interiors, Vol. 25, 297-356.

4. Neyman, Yr. (1979) Variational method of Physical Geodesy, Nedra, Moscow, (in Russian).

5. Marchenko, A.N., and Lelgemann, D. (1997). On the parameterization of the global Earth's models. Paper presented at the "Geodetic Week", Berlin, October, 1997.

6. Moritz, H. (1990) The Figure of the Earth. Theoretical Geodesy and Earth's Interior, Wichmann, Karlsruhe, 1990. 